\title{
Calendar of Events
}

American Physical Therapy Association

Combined Sections Meeting (CSM)

February 4-7, 2015

Indianapolis, Indiana

website: www.apta.org/csm

Arthroscopy Association of North America (AANA)

Metcalf/AANA Arthroscopic Surgery Seminar

\section{March 5-8, 2015}

Snowbird, Utah

website: www.posna.org/meetings/anmeet/anmeet.asp

$\bullet$

Association of Academic Physiatrists

2015 AAP Annual Meeting

March 10-14, 2015

San Antonio, Texas

website: www.physiatry.org/?page=meeting_2015

American Occupational Therapy Association

$95^{\text {th }}$ Annual Conference \& Expo

April 16-19, 2015

Nashville, Tennessee

website: www.aota.org/ConfandEvents.aspx
American Academy of Neurology

66th Annual Meeting

April 18-25, 2015

Washington, District of Columbia

website: www.aan.com/go/home

The Pediatric Orthopedic Society of North America

2014 POSNA Annual Meeting

\section{April 29-May 2, 2015}

Marriot Marquis

Atlanta, Georgia

website: www.posna.org

American Urological Association

AUA Annual Meeting

May 15-19, 2015

New Orleans, Louisiana

website: www.aua2015.org

European Academy of Childhood Disability

$27^{\text {th }}$ Annual Meeting of the European Academy of Childhood Disability

May 27-30, 2015

Copenhagen, Denmark

website: www.eacd2015.org 
Congress of Neurological Surgeons Annual Meeting

September 26-30, 2015

New Orleans, Louisiana

website: http://w3.cns.org/meetings/2014/index.asp

American Society of Hand Therapists (ASHT)

ASHT's 38th Annual Meeting

October 8-11, 2015

Sheraton Denver Downtown Hotel

Denver, Colorado

website: www.asht.org/education/asht-annual-meeting
The American Academy of Cerebral Palsy and Developmental Medicine (AACPDM) $69^{\text {th }}$ Annual Meeting

\section{October 21-24, 2015}

JW Marriott Austin

Austin, Texas

website: www.aacpdm.org/meetings/2015

American Academy of Pediatrics

AAP National Conference and Exhibition (NCE)

October 24-27, 2015

Washington, District of Columbia

website: http://www.aapexperience.org/ 